\title{
Microwave Breast Imaging: Time-Domain Experiments on Tissue Phantoms
}

\author{
Emily Porter*, Adam Santorelli, Mark Coates and Milica Popović \\ Department of Electrical Engineering and Computer Engineering \\ McGill University \\ Montreal, Canada \\ \{emily.porter, adam.santorelli\}@mail.mcgill.ca, \{mark.coates, milica.popovich\}@mcgill.ca
}

\begin{abstract}
This paper presents measurements performed with our time-domain system for microwave breast imaging. We demonstrate that, in the time-domain, skin does not decrease the tumor detection ability as compared to phantoms without skin. In fact, in certain cases a skin layer is shown to increase tumor response. The experimental set-up uses ultra-wideband antennas and tissue phantoms with electrical properties approximating those of actual tissue. Tumor detection experiments are conducted on two types of breast phantoms - simple ones containing only fat and malignant tissue, and more realistic ones, incorporating a thin layer of skin, fat and tumor, along with a matching medium between the skin and the antennas.
\end{abstract}

Keywords-microwave imaging; breast cancer detection; tissue phantoms

\section{INTRODUCTION}

Microwave imaging is currently being researched as a potential technique for detecting cancerous tissues within the breast. It is based on the innate contrast in the dielectric properties of healthy and malignant tissue, which is particularly evident over the microwave frequency range. Microwave breast imaging could be used alongside standard detection methods such as mammography, or magnetic resonance imaging (MRI) to increase the overall tumor detection ability. It offers two clear advantages over the standard techniques, namely that it would cause minimal discomfort to the patient and does not involve ionizing radiation [1], [2].

In this paper, we apply our novel microwave imaging system described in [3] to two types of tissue phantoms in order to observe the effect that the skin layer has on tumor detection. We show, for the first time, measurements in the time-domain demonstrating that the skin does not necessarily produce a negative effect on obtaining the tumor response. The idea behind the system is as follows. When a patient visits the doctor and obtains a clean bill of health, the microwave imaging system can be used on the patient to obtain a measured signal response of his or her "healthy" breast (this is called the baseline signal). After the initial breast scan, our proposed system can be used at prescribed intervals of time to detect if any abnormal tissue growth is occurring within the breast. In this way, it could provide an early warning system for breast cancer. Similarly, it could be used to monitor the changes of an already existing tumor or to check if chemotherapy or other treatments are having an effect.

The work presented here focuses on the comparison between tumor detection on a phantom with skin and a simple one without skin. By analyzing the results, we hope to determine if the presence of skin does have a significant effect on the tumor response and, if so, to identify antenna arrangements favorable for detecting the tumor response. In the following section, the time-domain set-up is first presented. Next, the experimental parameters, including the antenna configuration and breast phantom composition, are given. Finally, measurement results comparing the tumor response for phantoms with and without skin are shown.

\section{EXPERIMENTAL SYSTEM}

\section{A. Time-Domain Microwave Imaging System}

The experimental system is presented in this section. We use two antennas (Traveling Wave Tapered and Loaded Transmission Line Antennas, described in [3], [4]); one to transmit a wideband pulse and the other to receive the scattered wave after it has passed through the breast phantom. The antennas are designed specifically for use in microwave breast imaging, and thus have appropriately small size and are well matched to the breast tissue's average relativity permittivity (minimizing the return loss). The antennas are held in place by a radome, which surrounds the breast phantom on one side and has slots for the antennas on its other side. The radome is made of Alumina (Friatec [5]), and has a relative permittivity $\varepsilon_{\mathrm{r}}=9.6$. A drawing of the radome with dimensions noted is shown in Fig. 1, along with a photograph. The antenna slots carved into the exterior curve of the radome alternate in direction on the radome surfaces of $0^{\circ}$ and $180^{\circ}$, while along the surfaces of $90^{\circ}$ and $270^{\circ}$ all of the slots are in the same direction. As seen in the figure, there is space for 16 antennas; however, the set of measurements presented here used only two slots at a time (two antennas).

The breast phantoms are made of up to three separate tissue phantoms - fat, skin and tumor - combined into a single heterogeneous phantom. Each of the tissue phantoms has been carefully engineered to have dielectric properties (conductivity, relative permittivity) that approximate those reported for the 

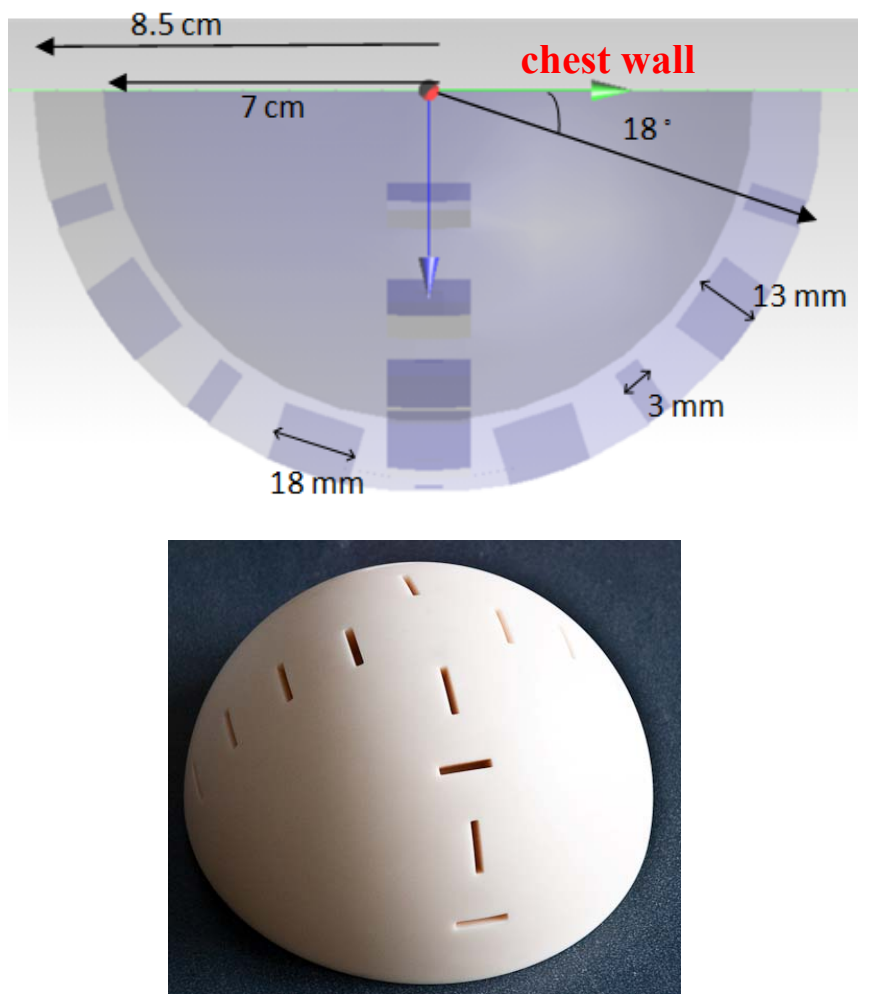

Figure 1. Drawing (top) and photograph (bottom) of the radome.

actual tissues. The procedures for making each tissue phantom and the complete breast model are detailed in [6].

For any desired set of measurements, the breast phantom is placed inside the radome and the transmitting and receiving antennas are each positioned in one of the radome slots. An impulse generator (Picosecond Pulse Labs, [7], Impulse Generator Model 3600) sends a pulse of duration $70-100 \mathrm{ps}$ with amplitude of $-7.5 \mathrm{~V}$ to the transmitting antenna. The pulse is transmitted at a repetition rate of $250 \mathrm{MHz}$, clocked by a Tektronix [8] gigaBERT 1400 generator. The pulse leaving the antenna propagates through the radome and into the breast phantom, where it is attenuated and scattered at each interface of different tissues or materials. The resulting wave is collected by the receiving antenna, and fed into a picoscope (Pico Technology, [9], PC Oscilloscope 9000), which is also being clocked by the gigaBERT. The picoscope connects into a computer, allowing the received data to be analyzed digitally.

\section{B. Experimental Parameters}

In this particular set of experiments, two distinct types of breast phantoms were used. The first is the most simple: the phantom is made entirely of fat-mimicking tissue, with one tumor of a given size and location. The more complex, and thus more realistic, version of the breast phantom has a $2.5-\mathrm{mm}$ layer of skin surrounding the fat. Also in this type, in between the skin and the radome is a roughly $0.5-\mathrm{cm}$ matching medium intended to reduce the mismatch between the antennas and the phantom, and to eliminate any air gaps between the radome and the breast phantom. The matching medium used is equivalent to the fat-mimicking material. All phantoms were made less than a week prior to the experiments.

For each phantom type, the tumor is approximately cylindrical with a height of $3 \mathrm{~cm}$ and $1 \mathrm{~cm}$ in diameter (the tumors are cut by hand and thus have rough surfaces). In addition to the tumor size, there are two other key parameters: the tumor location within the breast phantom and the position of the receiving antenna relative to the transmitting antenna. In each measurement, all three of these parameters are kept constant and every measurement is done with both types of phantoms for ease of comparison.

To obtain the tumor response, two measurements must be performed. First, a baseline measurement is recorded. This gives the response of the breast phantom in the absence of tumor tissue. Then, a tumor phantom is placed within the breast model and another measurement is taken. The tumor response is then calculated as the difference between the received signal with the tumor in place and the baseline.

Four measurement scenarios are considered: Cases A - D, described in Table I. A diagram of the set-up is also shown, as a view looking into the phantom and radome from the chest wall. The terms ' $\mathrm{t} x$ ' and ' $\mathrm{rx}$ ' denote the transmitting and receiving antennas, respectively. The tumor location, halfway between the radome centre and one of the antennas, is shown as a brown circle within the phantom area. For each case, the measurement is repeated for both phantom types. In Cases A and $\mathrm{B}$ the received signals represent the portion of the transmitted signal that has travelled all the way through the breast phantom. In Cases $\mathrm{C}$ and $\mathrm{D}$, we look at the backscattered signals.

\section{MEASUREMENT RESUlTS}

Table II presents the maximum received signal amplitudes and maximum tumor responses for Cases A - D. The measurement results are given for both the breast phantom with no skin and the phantom that has a thin skin layer.

As an example of the received signals, one complete period for Case A is shown in Fig. 2. The received signals for the phantom with skin, and for the phantom without skin, are plotted. Fig. 3 shows the tumor responses for both phantom types obtained using the Case A scenario. Similarly, Fig. 4 gives a plot of the received signals for both phantoms using the set-up described by Case D. Further, Fig. 5 shows the corresponding calculated tumor responses for Case D.

\section{DISCUSSION}

Before having carried out this set of experiments, we would have predicted that the received signals for a phantom with skin would be lower than for the same set-up with a phantom without skin in scenarios A and B. This is because the skin layer adds extra attenuation to the signal, and the extra interfaces (matching medium-skin and skin-fat) increase the number of material mismatches, each of which causes part of the wave to reflect while only a portion of it continues to travel ahead. For the same reason, we would also predict that in these cases (A and B) the tumor response with skin present would have a smaller amplitude than the tumor response without skin. 
TABLE I. DESCRIPTIONS AND DIAGRAMS FOR EACH CASE

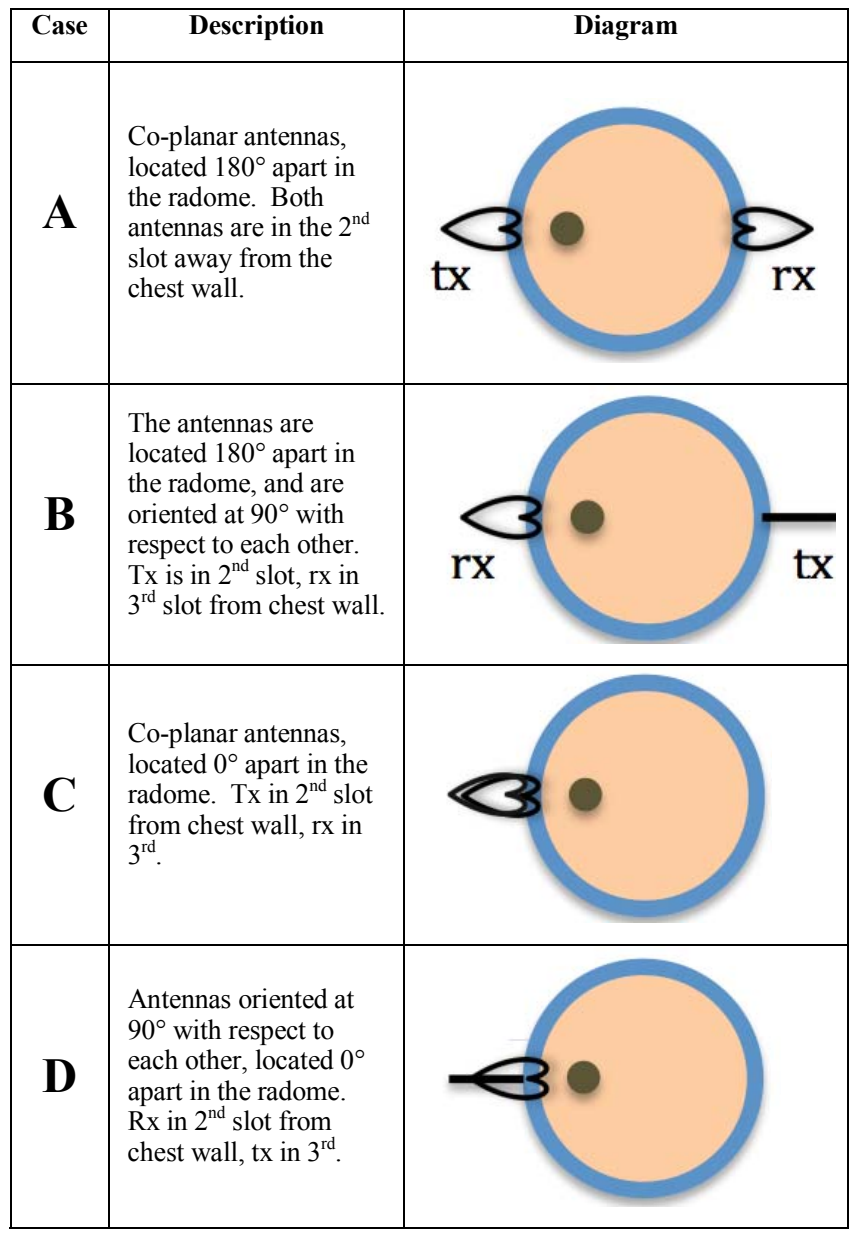

TABLE II. MAXIMUM RECEIVED SIGNALS AND TUMOR RESPONSES FOR EACH CASE USING BOTH PHANTOMS WITH AND WITHOUT A SKIN LAYER

\begin{tabular}{|c|c|c|c|c|}
\hline \multirow{2}{*}{ Case } & \multicolumn{2}{|c|}{ Received signal (mV) } & \multicolumn{2}{c|}{ Tumor response (mV) } \\
\cline { 2 - 5 } & No skin & With skin & No skin & With skin \\
\hline A & 18.8 & 43.5 & 11.4 & 3.70 \\
\hline B & 4.67 & 6.77 & 1.48 & 3.56 \\
\hline C & 113 & 121 & 45.9 & 11.8 \\
\hline D & 48.4 & 38.2 & 6.99 & 27.1 \\
\hline
\end{tabular}

Likewise, when the reflected signal is measured, as in Cases $\mathrm{C}$ and $\mathrm{D}$, we expected the received signal for the phantom with skin to be larger than without skin due to the large skin reflection being directly incident on the receiving antenna. We anticipated that the tumor response would be less for the phantoms with skin than without, since a relatively large portion of the signal is reflected off the skin leaving only a small amount of the initial wave energy to reflect at the fattumor boundary.

Our experiments have shown that these hypotheses are not generally true. In fact, depending on the antenna arrangement, the exact opposite can occur. First, we will discuss the transmission scenarios: Cases A and B. As shown in Table II,

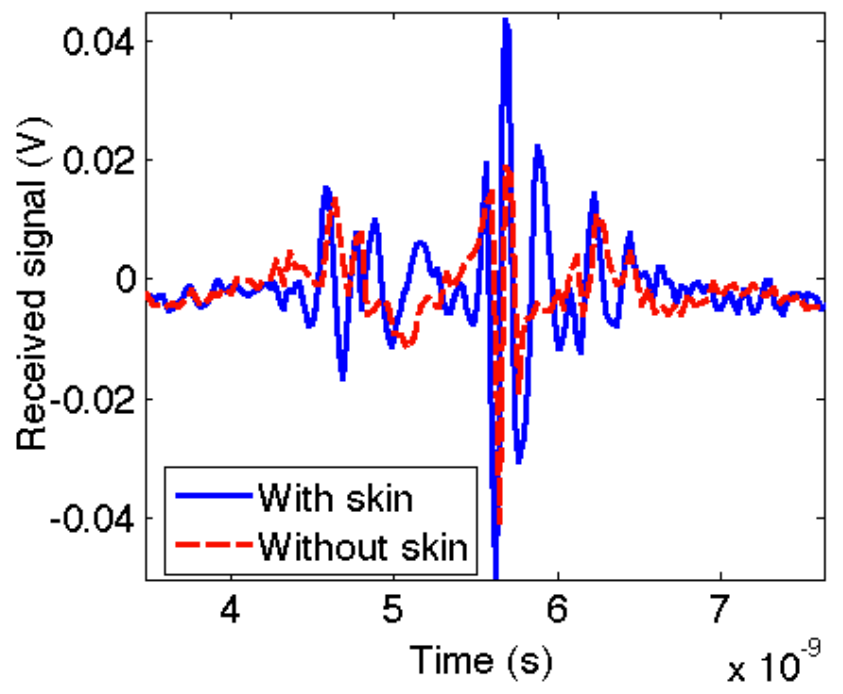

Figure 2. The received signal for Case A, plotted for both phantom types.

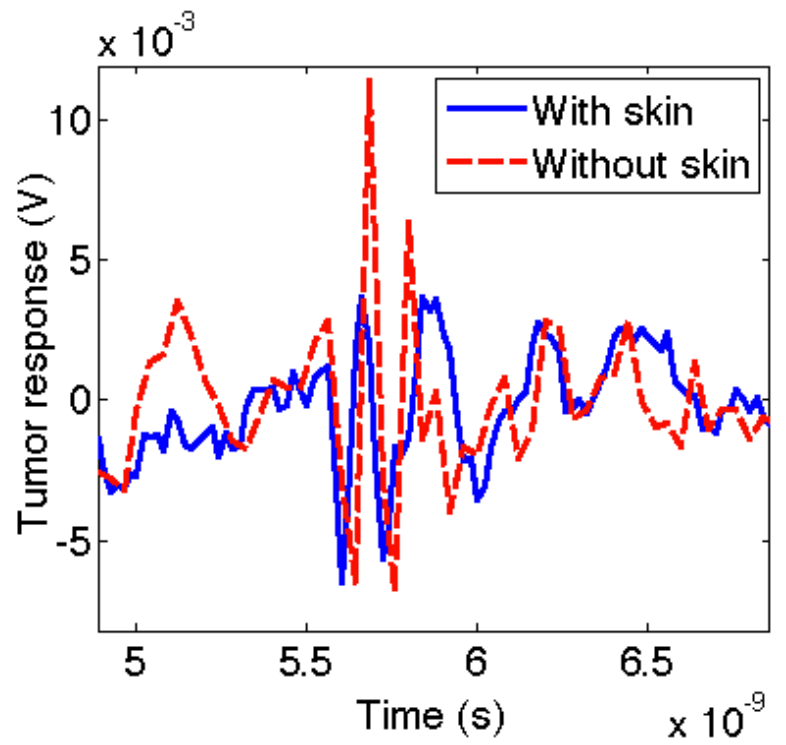

Figure 3. The tumor response for Case A, plotted for both phantom types.

in both cases the received signal with skin is higher than without skin. In Case A, the tumor response is three times smaller when skin is present relative to the case with no skin, while in Case B the tumor response is more than twice as large with skin.

As for the reflection measurements, Case C's received signal amplitude is only $7 \mathrm{mV}$ larger when the phantom has skin as compared to no skin, while the tumor response is 34 $\mathrm{mV}$ less with skin. In Case $\mathrm{D}$, on the other hand, the received signal is $10 \mathrm{mV}$ less and the tumor response is almost four times better when the phantom has skin.

Having examined the measurement results, only Case $\mathrm{C}$ fits with the original expectations. It has larger received amplitude for a phantom with skin than without, and a smaller tumor response. If we instead class the cases by the type of antenna arrangement, more information can be inferred. In Cases A 
and $\mathrm{C}$, the transmitter and receiver are oriented the same way. Alternatively, Cases B and D both use a receiver that is oriented at $90^{\circ}$ compared to the transmitting antenna. Focusing on the tumor responses, which for the purposes of breast cancer detection is a much more relevant metric than received signal amplitude, a correlation becomes apparent. In both cases where the antennas are similarly oriented (A and $\mathrm{C}$ ), the tumor response is better for a tumor located in a purely fat phantom than in a phantom with skin. When the antennas are perpendicularly oriented ( $\mathrm{B}$ and $\mathrm{D})$, the tumor response is higher when the phantom has a layer of skin.

The results show that perpendicularly oriented antennas, implying mutually perpendicular polarizations, obtained a better tumor response than do similarly oriented antennas when the phantom has skin. Since skin is an integral part of a realistic breast model, this result allows us to build a smarter antenna array for future experimentation.

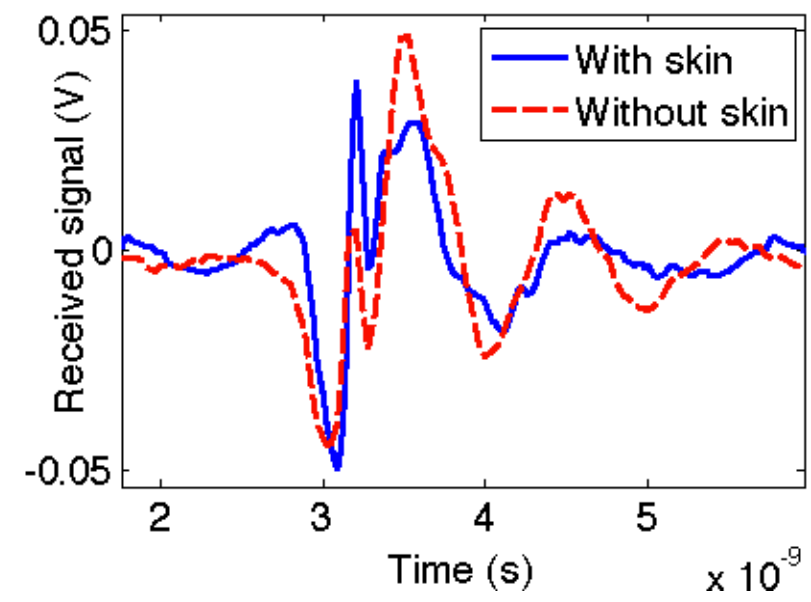

Figure 4. The received signal for Case D, plotted for both phantom types.

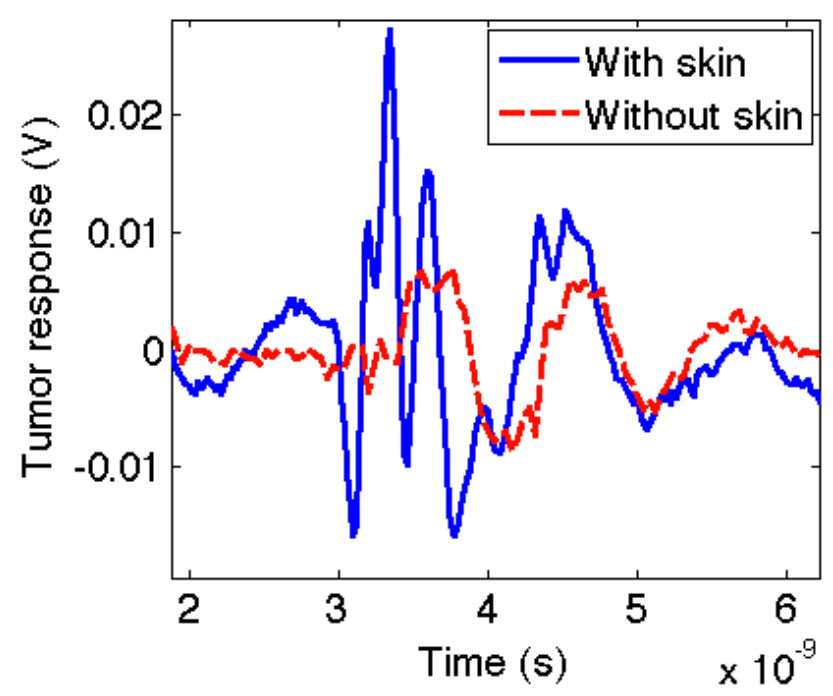

Figure 5. The tumor response for Case D, plotted for both phantom types.
Due to space constraints, only a limited number of cases are presented in this paper. However, a total of 39 measurement scenarios (listed in [3] for a phantom with skin) were made for each phantom type and analyzed in the same manner as explained for Cases A - D. We note that for all of our measurements, for different antenna positions and tumor sizes and locations, the trends observed here hold true.

The reason why perpendicularly oriented antennas provide better results than antennas parallel to each other may lie in the polarization. The wave polarization may be altered by the tumor so that it no longer matches the polarization of the transmitting antenna nor the receiving antenna when it is oriented similarly to the transmitter.

\section{CONCLUSION}

This paper has presented a comparison between experimentally measured tumor responses of breast phantoms with and without skin. It has shown that contrary to the hypothesis, the skin layer does not necessarily hinder the ability to receive the tumor response, and in some cases skin even increases the amplitude of the tumor response. We have also seen that the tumor responses are improved for perpendicularly oriented antennas as compared to those obtained when the antennas are parallel to each other, and this fact will be taken into consideration when designing the antenna arrays of future experiments. We also intend to investigate further the various antenna orientations to identify why the perpendicular arrangement leads to a better tumor response.

\section{REFERENCES}

[1] E.C. Fear, P.M. Meaney and M.A. Stuchly, "Microwaves for breast cancer detection?" IEEE Potentials, pp. 12-18, February/March 2003.

[2] I.J. Craddock, A. Preece, J. Leendertz, M. Klemm, R. Nilavalan and R. Benjamin, "Development of a hemi-spherical wideband antenna array for breast cancer imaging," European Conference on Antennas and Propagation EUCAP 2006, Nice, France, 6-10 Novemeber 2006.

[3] E. Porter, A. Santorelli, M. Coates and Milica Popović, "An experimental system for time-domain microwave breast imaging," European Conference on Antennas and Propagation EUCAP 2011, Rome, Italy, 11-15 April 2011, in press.

[4] H. Kanj and M. Popović, "A novel ultra-compact broadband antenna for microwave breast tumor detection," Progress in Electromagnetics Research, vol. 86, pp. 169-198, 2008.

[5] Friatec: http://www.friatec.com/

[6] E. Porter, J. Fakhoury, R. Oprisor, M. Coates and M. Popović, "Improved tissue phantoms for experimental validation of microwave breast cancer detection," European Conference on Antennas and Propagation EUCAP 2010, Barcelona, Spain, 12-16 April 2010.

[7] Picosecond Pulse Labs: http://www.picosecond.com/

[8] Tektronix: http://www.tek.com/

[9] Pico Technology: http://www.picotech.com/ 